\title{
IMPORTÂNCIA DO ESTUDO DA FUNÇÃO DA SENSIBILIDADE AO CONTRASTE NO GLAUCOMA
}

\author{
IMPORTANCE OF THE STUDY OF THE CONTRAST SENSITIVITY FUNCTION IN GLAUCOMA
}

Antonio C. Silva ${ }^{1} \&$ Maria de Lourdes Veronese Rodrigues ${ }^{2}$

${ }^{1}$ Oftalmologista. Departamento Médico da Câmara dos Deputados. Doutor em Oftalmologia. ${ }^{2}$ Docente. Faculdade de Medicina de Ribeirão Preto - USP.

CoRRESPONDÊNCIA: Antonio C. Silva. Quadra 205, Lote 08, Apto.601. CEP 72030-100 Brasília-DF. Email:antocarva@medico-df.com.br

SILVA AC \& RODRIGUES MLV. Importância do estudo da função da sensibilidade ao contraste no glaucoma. Medicina, Ribeirão Preto, 35: 497-504, out./dez. 2002.

RESUMO: Considerando-se que as aferições da Acuidade Visual (AV) com os optotipos de Snellen não correspondem ao real poder da visão, desde a década de 1980, vêm os estudiosos pesquisando novas formas de avaliar o fenômeno, culminando com a aplicação da Função da Sensibilidade ao Contraste (FCS), um exame psicofísico, de inestimável valor no estabelecimento do diagnóstico precoce de inúmeras doenças.

Entretanto, apesar do interesse despertado e dos valores relativos a sua sensibilidade e especificidade, o estudo da FSC não teve a utilização que seria de se esperar, na rotina oftalmológica, devido a resultados que, a princípio, parecem conflitantes.

A despeito disso, para os autores deste artigo, as aparentes discrepâncias são devidas a diferentes formas de abordagem do exame, cuja aplicação não tem obedecido a uma necessária padronização metodológica, e acreditam que, tão logo haja uma uniformização de avaliação da FSC, os resultados passarão a ser coerentes e sua plena aceitação será um fato.

Além do grande valor no diagnóstico precoce de inúmeras doenças, a FSC também poderá ser muito útil na Medicina do Trabalho e na avaliação das condições psicofísicas de candidatos à obtenção de autorização para conduzir veículos automotores terrestres, marítimos e aéreos, podendo ser um fator limitante na habilitação dessas pessoas.

UNITERMOS: Sensibilidades de Contraste. Glaucoma. Hipertensão Ocular.

\section{INTRODUÇÃO}

O ano 2002 constituiu-se em momento ímpar para a Oftalmologia mundial, pelo fato de nele ter acontecido a comemoração dos 140 anos de edição da escala de optotipos de Snellen, criada para mensurar a Acuidade Visual (AV) - o poder de resolução da visão, capaz de distinguir a menor distância entre dois pontos contíguos.

Entretanto, apesar do uso generalizado dessa escala, é sabido que a AV, tal como tem sido praticada até hoje, não tem o rigor científico, necessário para sustentar uma definição indiscutível do que seja, de fato, a capacidade da visão.

Uma definição moderna e atualizada de acuidade visual faz referência ao poder de resolução, capaz de distinguir o mínimo ângulo de visão que duas barras adjacentes, caracterizadas por intensidades luminosas diferentes, possam ser percebidas no nível dos receptores maculares, e inclui ainda a cor, o movimento e a sensibilidade ao contraste (SC) espacial e temporal $^{(1,2,3)}$. 
$\mathrm{Na}$ avaliação da Função da Sensibilidade ao Contraste (FSC), o padrão espacial é composto de barras claras e escuras que, alternando-se dentro do espaço de um ângulo de visão, constitui a freqüência espacial, enquanto o padrão temporal é composto por uma fonte de luz que pisca seqüencialmente (flicker) até se fixar, por alguns instantes, em uma unidade de tempo, o que vem a se constituir na freqüência temporal ${ }^{(1)}$. O número de barras claras e escuras é estabelecido de acordo com a freqüência espacial, que corresponde ao número de ciclos do padrão ( 1 ciclo $=1$ barra clara +1 barra escura), por grau de ângulo de visão.

Estudos neurofisiológicos têm suportado a noção de que o sistema visual utiliza-se de uma forma particular de análise da frequiência espacial no processamento primário da informação visual ${ }^{(4)}$.

Tais constatações psicofísicas levaram ao estudo da FSC, aprofundando o conhecimento sobre o estado e a sensibilidade dos receptores retinianos, fornecendo, assim, uma informação de caráter qualitativo, enquanto a AV avaliada com os optotipos de Snellen fornece uma informação de caráter quantitativo ${ }^{(3)}$.

\section{MATERIAL E MÉTODOS}

Os estudos iniciais dessa nova metodologia datam de 1956 e incluem conhecimentos prévios de Matemática, Geometria, Física Óptica ${ }^{(5)}$ e conceitos adaptados da Engenharia de Análise de Sistemas ${ }^{(6)}$, da Fisiologia e da Fisiopatologia ${ }^{(2)}$, tendo sido imprescindíveis na definição dos componentes ópticos e neurais, que fundamentam o estudo da função da sensibilidade ao contraste ${ }^{(4)}$.

Talvez, a melhor e mais adequada definição de sensibilidade ao contraste espacial seja dada pela sua curva, que é composta do registro de pontos que representam os mínimos contrastes perceptíveis, em função da frequiência espacial. Essa curva é expressa por um sistema cartesiano, no qual, sobre o eixo das ordenadas, insere-se o inverso do contraste, expresso em percentuais do contraste máximo (100\%), e sobre o eixo das abscissas, insere-se a frequiência espacial, medida em ciclos por grau (c/g).

O valor do contraste é calculado segundo a fórmula:

$\mathrm{C}=(\mathrm{L} \max -\mathrm{L} \min ) /(\mathrm{L} \max +\mathrm{L} \min ) X \mathbf{1 0 0}$, onde $\mathbf{C}$ é o contraste;

Lmin é a luminosidade mínima;

Lmax é a luminosidade máxima; e

100 é o máximo percentual do contraste.
Para realização dos exames, utiliza-se um computador IBM compatível, no qual, uma placa gráfica do tipo super vídeo Graph Adapter (VGA), modificada, permite obter, de um monitor de TV monocromático, também super VGA, com poder de resolução de 480 linhas X 650 pontos, 4.096 tonalidades diferentes do cinza ${ }^{(3)}$.

\section{RESULTADOS}

Inúmeras são as variáveis que interferem no desempenho do olho submetido aos testes de sensibilidade ao contraste. Dentre as mais comuns, podem ser incluídos o vício de refração, a ambliopia, a idade do paciente, a adaptação ambiental ${ }^{(7)}$ a percepção de cores e o diâmetro pupilar ${ }^{(1)}$. Podem, ainda, ser relacionados o uso de lentes de contato-com e sem filtro UVA/UVB ${ }^{(8)}$ - a catarata, as opacidades corneais, as doenças do nervo óptico, o glaucoma ${ }^{(9)}$, a esclerose múltipla $^{(10)}$, a doença macular relacionada à idade (DMRI), o mal de Parkinson, o diabetes mellitus (DM), o tumor hipofisário ou pancreático ${ }^{(11)}$, a manipulação de agrotóxicos ${ }^{(12)}$, o cimento, o amianto, a sílica ou os efeitos colaterais de medicamentos ${ }^{(13)}$.

Observações clínicas e laboratoriais têm fornecido informações no sentido de que o resultado das curvas da FSC é muito diferente, quando se analisam os dados de um paciente sadio (avaliado como normal) com os de outro que, embora em condições homeostáticas, seja portador de uma determinada doença. Por esse motivo, é razoável admitir que a utilização do teste da FSC é capaz de revelar, precocemente, distúrbios do sistema visual, que não são revelados pela avaliação tradicional com os optotipos de Snellen ${ }^{(4)}$

\section{DISCUSSÃO}

Para que o sistema visual possa distinguir um padrão composto por barras alternadas (claras e escuras), com dimensões, formas e luminosidades diferentes, de modo indiscutível, é necessário que os componentes da freqüência Fourier estejam acima de um valor limite e que o espectro de sua curva tenha forma quadrada ou senoidal $\left.{ }^{(6,14}\right)$.

Tipicamente, a extensão da freqüência espacial em uma determinada curva da FSC está entre $0,5 \mathrm{e}$ $30 \mathrm{c} / \mathrm{g}$. A $30 \mathrm{c} / \mathrm{g}$, o número de barras claras e escuras corresponde a 1,0' (um minuto de arco), que é igual ao tamanho do optotipo 20/20, da escala de Snellen ${ }^{(15)}$. Nessa situação, o espectro da curva de sensibilidade 
ao contraste versus a frequiência espacial em ciclos por grau, caracteristicamente, apresenta um pico nas frequiências médio-baixas, com redução da sensibilidade para valores abaixo ou acima dessa faixa. No limite de alta frequiência, a $30 \mathrm{c} / \mathrm{g}$, quando o contraste requerido é de $100 \%$, a medida da acuidade visual corresponde a 20/20 da escala de Snellen e os vícios de refração assumem, portanto, substancial importância entre 5 e $12 \mathrm{c} / \mathrm{g}(4,14,16,17)$.

Alguns estudos sugerem que a mensuração da FCS é o equivalente visual de um audiograma e essa analogia tem grande utilidade, particularmente, porque, no âmbito da freqüência espacial, existem muitos paralelos entre o processamento auditivo e o visual.

Sabendo-se que a atividade cerebral realiza análises semelhantes às dos componentes da freqüência Fourier, as alterações dessa freqüência são o modo de o sistema visual analisar, transmitir e reconstruir a imagem retiniana.

Evidências experimentais, em gatos, mostraram que diferentes canais existentes na retina, provenientes do corpo geniculado lateral e do córtex, conduzem informações apropriadas, relacionadas com diferentes frequiências espaciais. Também tem sido realçado que todos os canais respondem ao contraste, com o córtex revelando, de fato, uma conexão linear entre a amplitude da descarga neuronal e o logaritmo do contraste da grade senoidal ${ }^{(18)}$. Isto é, há evidências neurofisiológicas e psicofísicas de que a informação espacial é processada pelos canais neurais, que são sintonizados com a freqüência espacial ${ }^{(4,15)}$.

Assim, foi sugerido que o termo sensibilidade ao contraste é suficientemente útil para ser chamado de visuograma ${ }^{(2)}$.

Vários estudos têm demonstrado que, na fisiopatologia do glaucoma primário, de ângulo aberto, as visões paracentral e periférica estão comprometidas bem antes que a visão macular seja reduzida. Entretanto, essa evidência de que a visão central só é afetada tardiamente, tem sido baseada apenas na aferição da visão, feita através do uso de escalas de sinais e letras do tipo Snellen, como único método de avaliação da visão central. Sabe-se que o teste de Snellen mede apenas o poder resolutivo do olho para valores próximos do contraste máximo e ignora parâmetros, como a percepção de cores, a habilidade para detectar objetos de baixo contraste, a percepção de movimentos, a percepção de formas e a distinção de objetos sob baixa iluminação, que deixam de ser avaliados adequadamente $^{(1,14)}$.
Em um estudo com 23 pacientes, onde apenas 5 eram suspeitos de ser portadores de glaucoma, ficou demonstrado que mais de $50 \%$ das fibras do nervo óptico estavam lesadas, nos casos de glaucoma, antes que qualquer anormalidade pudesse ser detectada pela perimetria cinética de Goldmann ou pelos optotipos de Snellen. Desse modo, tanto o pólo superior quanto o pólo inferior do nervo óptico tinham grande destruição das fibras nervosas, arqueadas em toda a sua extensão. Além disso, esse estudo também estabeleceu que, com a evolução do glaucoma, a densidade da camada de fibras nervosas sofria redução em toda a extensão do nervo óptico, até que pudesse vir a ser detectada como restrição de campo visual periférico. Assim, apesar do pequeno número de pacientes estudados, os autores demonstraram evidências histopatológicas de que as lesões das fibras do nervo óptico, no glaucoma, são processadas em duas vias: 1) focalmente, nos pólos superior e inferior do nervo e 2) difusamente, em toda a extensão da população das fibras nervosas. Essa conclusão foi confirmada, posteriormente, por outros estudos, que demonstraram perdas focais, difusas e mistas na camada de fibras nervosas, através de fotografias do fundo do olho glaucomatoso. Ademais, o glaucoma também pode produzir danos morfológicos na camada de fibras nervosas, focalmente, difusamente ou associando as duas formas ${ }^{(1,14)}$.

Estudos diversos, de vários autores, demonstraram defeitos adquiridos na percepção de cores no glaucoma. Entretanto, os estudos relacionados com a percepção de cores são muito difíceis de comparação, por causa das diferentes metodologias empregadas. Todavia, muitos desses trabalhos suportam o conceito de que o mecanismo azul-amarelo, em geral, é mais severamente afetado, no glaucoma, do que o mecanismo vermelho-verde ${ }^{(1)}$. Alguns pesquisadores não conseguiram demonstrar significante correlação entre pacientes suspeitos ou portadores de glaucoma primário de ângulo aberto e percepção anormal de cores, quando utilizaram o Farnsworth-Munsel 100 Hue Test, talvez pelas dificuldades acima descritas.

A despeito desses estudos, a maioria dos trabalhos publicados indicam que a visão de cores pode estar afetada, precocemente, no processo glaucomatoso, muitas vezes, antes que outros distúrbios visuais possam ser detectados ${ }^{(1)}$.

Há uma significante correlação entre a perda da visão de cores e o decréscimo de sensibilidade à luz, na zona central, paracentral e na média periferia da retina. 
Desse modo, pacientes com alterações precoces de campo visual devido a glaucoma crônico têm defeito no processamento das vias ópticas tanto para cores quanto para luzes, nos dois graus centrais do campo visual.

Além disso, uma vez que essas duas vias ópticas são mediadas por populações distintas de células ganglionares pequenas $(\mathrm{X})$ e grandes $(\mathrm{Y})$, ambos os tipos são $\operatorname{afetados}^{(10,14,19)}$.

Bron reconheceu que o sistema visual é organizado de modo a distinguir elementos espaciais e temporais em variados níveis de contraste, e que essa habilidade depende de subsistemas neurais, que incluem células ganglionares $\mathrm{X}$ e $\mathrm{Y}$, da retina. As células $\mathrm{X}$ têm alta sensibilidade ao contraste espacial, porém, baixa sensibilidade ao contraste temporal. O oposto é verdadeiro para as células ganglionares Y. Como os pacientes glaucomatosos apresentam redução na sensibilidade ao contraste espacial e temporal, existem fortes evidências de que, nessa doença as células X e Y estejam comprometidas ${ }^{(20)}$.

A perda da FSC não é específica do glaucoma, uma vez que outras causas de decréscimo dessa função, como a catarata e a ambliopia, são comuns em pacientes glaucomatosos. Tais causas necessitam ser excluídas antes que conclusões apressadas possam ser extraídas acerca do teste de sensibilidade ao contraste. Significantes diferenças foram observadas em várias frequiências espaciais, quando de um controle não muito cuidadoso, relativo à idade e a possíveis alterações do cristalino. O teste da sensibilidade ao contraste espacial pode ser muito útil como exame diagnóstico complementar no glaucoma ou em outras patologias, porém, interpretar os resultados sem outros dados clínicos pode induzir a erros no diagnóstico definitivo $^{(1,13)}$.

Quanto à sensibilidade ao contraste temporal, alguns estudos sugerem que os resultados dos testes desse modo de avaliação da sensibilidade ao contraste separam os olhos glaucomatosos dos olhos normais, melhor do que os testes de sensibilidade ao contraste espacial $^{(19)}$.

Embora existam alguns trabalhos que ilustrem o contrário, as divergências podem realçar, apenas, a prática de diferentes metodologias (de testes e/ou definições clínicas).

Outros estudos, porém, não constataram diferenças substanciais entre os testes de sensibilidade ao contraste espacial ou temporal ${ }^{(19)}$.

Um aspecto a ser evidenciado é que o estudo das curvas da FSC deve ser realizado com a análise de estímulos estáticos e dinâmicos.

Os estímulos estáticos têm, em sua gênese, aqueles obtidos com barras quadradas, estanques, onde o preto finda abruptamente, exatamente onde se inicia o estímulo branco, e vice-versa, ou seja, o estímulo branco começa onde finda o preto, e assim, sucessivamente.

Do mesmo modo, os estímulos dinâmicos originam-se daqueles obtidos com barras senoidais, contínuas, onde não há um locus específico para findar o estímulo branco ou iniciar o estímulo preto, e viceversa.

Uma pesquisa, envolvendo 232 olhos, constatou que a sensibilidade ao contraste temporal parece ser determinada pela retina periférica, uma vez que a estimulação dessa área foi mais bem sucedida que nas demais, sugerindo que essa condição poderia ser usada para o diagnóstico do glaucoma inicial ${ }^{(21)}$.

A maior dificuldade de realização dos testes psicofísicos (portanto, testes subjetivos) é que os mesmos requerem a cooperação do paciente e nem todos são capazes para dar respostas confiáveis. Contudo, modernas técnicas de exame têm proporcionado respostas consistentes para a maioria dos pacientes e o grau de confiança elevou-se de modo a conferir o real valor dos resultados obtidos ${ }^{(17)}$.

Em avaliações pediátricas, algumas condições demonstraram uma resposta normal para o teste de acuidade visual do tipo Snellen, porém resultados anormais, quando testados o campo visual ou a função da sensibilidade ao contraste em uma variedade de doenças, como esclerose múltipla, diabetes mellitus, glaucoma, tumores do sistema nervoso central (SNC) e do sistema visual.

Entretanto, devido ao tempo necessário para conduzir o teste e o desafio em se obter uma resposta subjetiva consistente, a avaliação da sensibilidade ao contraste, em crianças, tem encontrado resistências na sua aplicação( ${ }^{(2)}$.

Além disso, muitos estudos chamam a atenção para o fato de que os testes de sensibilidade ao contraste são confusos em sua interpretação. Entretanto, entende-se que, muito mais que falta de compreensão, ocorre falta de uniformização de metodologias, caracterizada pelo uso indiscriminado de optotipos ou faixas alternadas pretas e brancas (impressas), de fonte de luz piscante (flickers), de monitores de TV com padrões diversos, de inobservância da necessária padronização da iluminação ambiente, de diferen- 


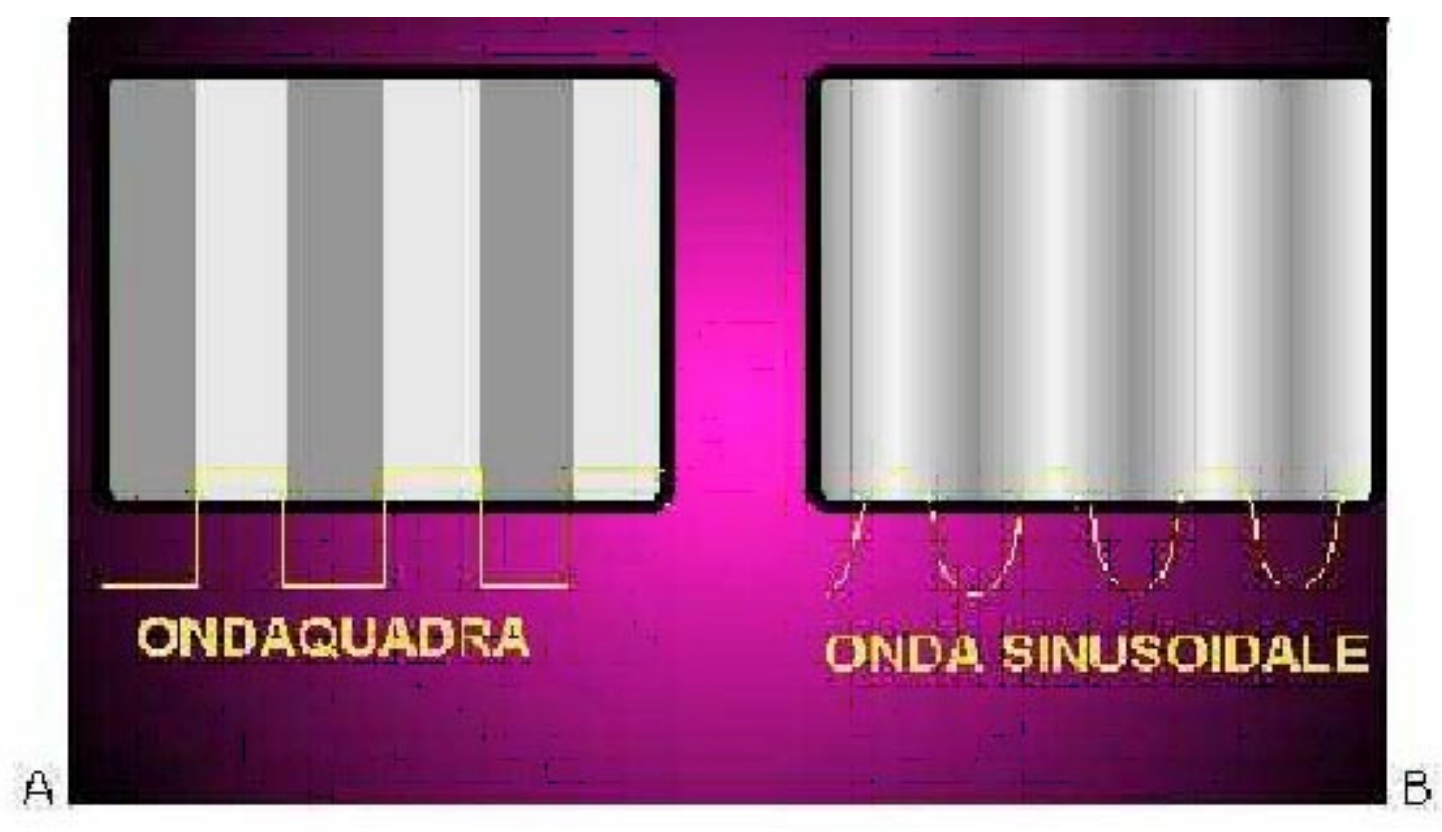

Figura 1: Estímulo com onda quadrada $(A=$ ondaquadra) e estímulo com onda senoidal $(B=$ onda sinusoidale) Imagens cedidas pelo Professor Doutor Eduardo Rispoli - Universitá Deqli Studi di Roma La Sapienza.

tes distâncias do paciente à fonte, ou ainda, do fato de o teste aplicado ser estático ou dinâmico. A esses aspectos, acrescenta-se a presença concomitante de outras moléstias, eventualmente presentes no paciente examinado, não levantadas na anamnese e a falta de curvas-padrão, características de cada patologia, para balizar a interpretação dos resultados obtidos.

É importante lembrar que esse teste mede a função do sistema visual como um todo (isto é, meios ópticos, retina e vias oculares superiores no SNC) e, antes que alguma conclusão possa ser extraída acerca do local da disfunção visual, deve ser considerada a contribuição potencial de outros elementos ${ }^{(17)}$.

$\mathrm{O}$ uso de testes de visão de cores da prática clínica [American Optical-Hardy-Ritter-Rand (AOHRR), color plates e Farnsworth D-15 panel], em 19 pacientes com glaucoma primário, de ângulo aberto, 19 pacientes com hipertensão intra-ocular sem danos de campo visual detectáveis, e 19 pacientes de um grupo-controle, todos com idades semelhantes, demonstrou um significante defeito na visão de cores, mais frequiente no grupo glaucomatoso e no grupo de hipertensos oculares, do que no grupo-controle, para aqueles testes $^{(15,20)}$.

Assim, para o teste Farnsworth D-15, obtiveram-se $78 \%$ de defeitos para os olhos glaucomatosos, $58 \%$ de defeitos para os olhos de hipertensos ocula- res e apenas $11 \%$ para os olhos de indivíduos do grupo-controle.

Entretanto, somente o seguimento do grupo de hipertensos oculares, ao longo do tempo, poderia estabelecer se esses pacientes, suspeitos de glaucoma, viriam a desenvolver perdas de campo visual ${ }^{(1,14)}$.

O desenvolvimento e a aplicação da metodologia da FSC poderiam proporcionar um novo e indiscutível conceito do que seja acuidade visual, bem como as alterações dessa acuidade forneceriam elementos imprescindíveis para firmar o diagnóstico de diferentes doenças através do conhecimento prévio das nuances de suas respectivas curvas, sempre ricas em cada um dos seus detalhes pontuais, nas respectivas freqüências espaciais [onde o contraste-limite é avaliado para cada uma das respectivas freqüências $\left.{ }^{(23)}\right]$.

Os achados que apresentamos estão em concordância com relatos da literatura de que o sistema visual é mais sensível nas frequiências espaciais intermediárias, que são constituídas de poucos ciclos por grau, e que é relativamente insensível para baixas e altas freqüências espaciais, como nos casos de lesões maculares e cerebrais, justificando, assim, uma análise objetiva do gráfico da FSC nessas freqüências e não em todo o espectro da curva, constituída, em geral, do registro de cinco ou mais freqüências) ${ }^{(6,14)}$. 


\section{CONCLUSÃO}

A FSC, como proposta de uma nova metodologia para o conhecimento preciso da acuidade visual, que tenha a mesma praticidade, viabilidade, confiabilidade, previsibilidade e reprodutibilidade como exame diagnóstico preciso e que, todavia, tenha a popularidade da escala de optotipos de Snellen é, hoje ainda, um grande desafio, uma utopia. Pois, em que pesem inúmeros trabalhos realizados nos mais respeitáveis centros de pesquisas de todo o mundo, os resultados não têm correspondido às expectativas iniciais, muito provavelmente porque tais pesquisas foram promovidas com distintos equipamentos, em condições ambientais e padronizações as mais diversas e, possivelmente, ainda, pelos altos custos que lhes são peculiares, principalmente quando comparados ao valor de uma cartela de optotipos, impressa em papelão.

É sabido que o princípio do estudo da sensibilidade ao contraste se baseia na observação de que, na própria natureza, os animais, das mais diversas espécies, se mimetizam para fugir dos predadores ou para caçar as suas presas. Esse mimetismo ou metamorfose é o mesmo princípio utilizado pelos militares, quando se camuflam, e aos seus equipamentos ou, ainda, por agricultores, pescadores e tantos outros segmentos que, por suas necessidades bélicas, profissionais ou esportivas precisam utilizar-se de um certo tipo de disfarce, de modo a despistar algo ou alguém. Isso, em síntese, representa o uso prático da redução do contraste.

Cientes da utilidade do conhecimento desse fenômeno, redobrados cuidados dever-se-iam tomar ao trafegar por estradas que cortam regiões de preservação ecológica da flora e da fauna, evitando-se, assim, por exemplo, o atropelamento e morte de animais, algumas vezes, até pertencentes a espécies raras e/ou ameaçadas de extinção.

A importância do estudo e aplicação da FSC também encontra guarida na prática de certas atividades profissionais, como na aviação, na pilotagem de um navio ou na segurança dos meios de transportes terrestres, particularmente no período da aurora ou do crepúsculo, quando se perde parcialmente, por algum tempo, a plena capacidade de distinguir, por exemplo, um automóvel preto se deslocando sobre uma pista de asfalto também preto.

A figura abaixo tenta demonstrar a expressão gráfica das afirmações citadas acima.

Desse modo, a FSC poderá, algum dia, ser utilizada como meio de avaliação de candidatos para o exercício de determinadas profissões e, ainda, como meio semiótico na Medicina do Trabalho.

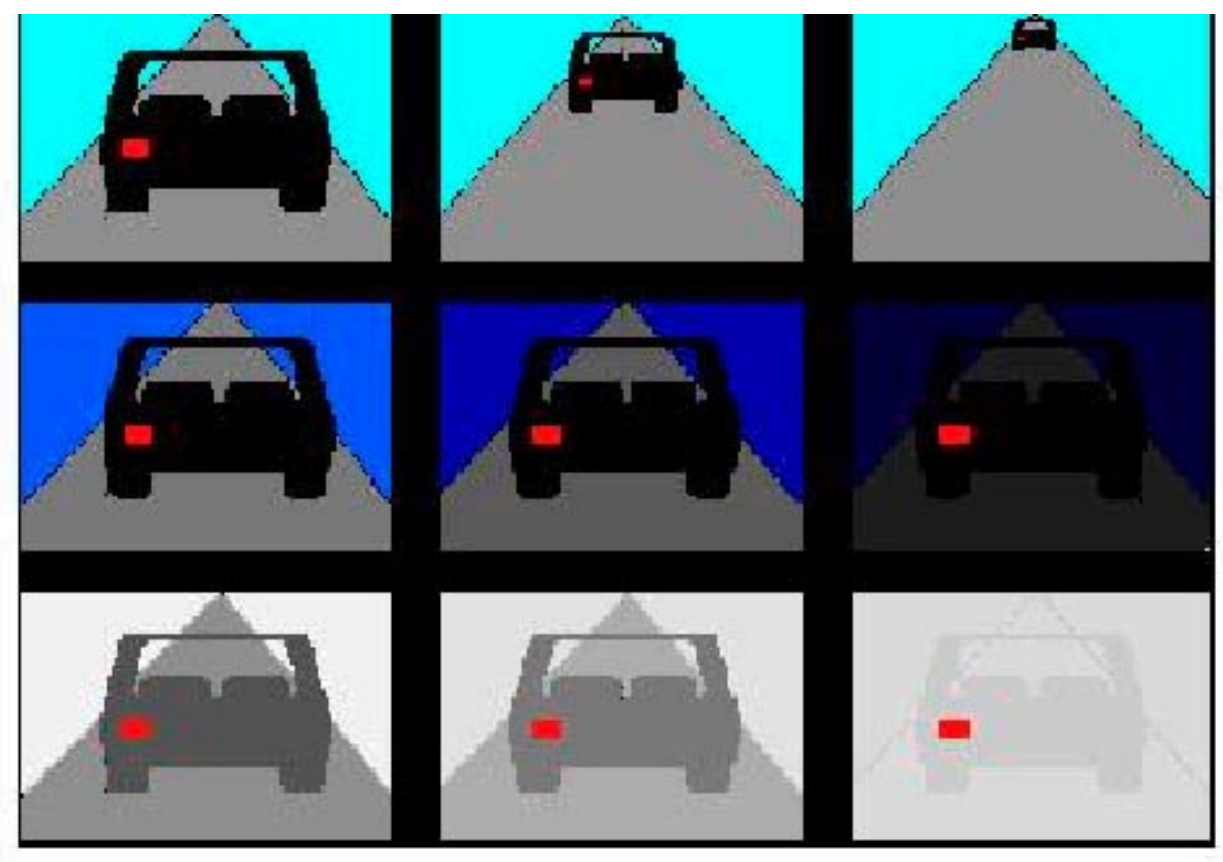

Figura 2: Exemplo de diferentes níveis de contraste. Imagem cedida pelo Professor Doutor Eduardo Rispoli - Universitá Degli Studi di Roma La Sapienza. 
SILVA AC \& RODRIGUES MLV. Importance of the study of the contrast sensitivity function in glaucoma. Medicina, Ribeirão Preto, 35: 497-504, oct./dec. 2002.

ABSTRACT: Considering that, the measurements of the Visual Acuity (VA) with the Snellen's letters do not correspond the reality of the power of the vision, since 1980, new forms to measure this phenomenon have been reserched. One of the possible solutions is the application of the Contrast Sensitivity Function (CSF) exam, which is a psicophisical test of a very importance value for the establishment of precocius diagnostic for several diseases.

In spite of the arisen interest about the exam and also the relative values of its sensibility and specificity, the study of the CSF hasn't been used the way it was expected in ophthalmologic routine due to apparently conflictive results.

For the authors of this article, although, most of the discrepances are apparently related to the distinctive ways of approaching the exam, whose application doesn't follow the necessary methodological design.

It's belived that as soon as a pattern is set for the valuation of the CSF exam, the results will no longer be incoherent and it's full acceptance will be a fact.

Besides the great value of the precocious diagnostic over several diseases, the CSF can also be extremely useful in the work medicine, measuring the conditions of the candidates on the acquisition of their document of habilitation for the conduction of maritimes, airyes and earthen vehicles. The exam on this case can be a limitant factor on the concession of this licenses.

UNITERMS: Contrast Sensitivity. Glaucoma. Ocular Hypertension.

\section{REFERÊNCIAS BIBLIOGRÁFICAS}

1 - STAMPER RL. Psycophysical changes in glaucoma. Surv Ophthalmol 33:309-318, 1989. Supplement.

2 - BODIS-WOLNER I. Visual acuity and contrast sensitivity in patients with cerebral lesions. Science 178:769-771, 1972.

3 - IPPOLITI G. Prime esperienze con un nuovo strumento per rilievo automatico della curva della sensibilitá al contrasto. Tesi di Specializzazione presentata alla II Scuola di Specializzazione in Oftalmologia dal Istituto di Oftalmologia - Clinica Oculistica della Facoltá di Medicina e Chirurgia della Universitá degli Studi di Roma "La Sapienza". Roma, Itália. p. 1-27, 1991.

4 - JOHNSON CA. Evaluation of visual funtion. Duane's: Foundations of clinical Ophthalmology. Lippincott-Raven, Philadelphia, cap. 17, p. 5-6, 1994.

5 - SCHADE OH. Optical and photoelectric analog of the eye. $\mathbf{J}$ Opt Soc Am 46:721-739, 1956.

6 - ENROTH-CUGELL C \& ROBSON JG. The contrast sensitivity of retinal ganglion cells of the cat. J Physiol 187: 517-552, 1966.

7 - SILVA AC. \& SANTOS EF. Pesquisa da sensibilidade ao contraste (SC). IN: RODRIGUES MLR \& DANTAS AM, eds. Oftalmologia clínica, 2ed., Cultura Médica, Rio de Janeiro, cap 5.3 p. 69-76, 2001.

8 - SILVA AC HU T; RISPOLI E; RAPAGNETTA L; LEONARDI A; MOAURO E \& DE LEO A. Utilizzazione del test della sensibilità al contrasto nello studio delle lenti a contatto con filtri ultravioletti. Boll Oculistica 2:365-373, 1993.
9 - TYTLA ME. Flicker sensitivity in treated ocular hypertension. Ophthalmology 97:36-43, 1990.

10 - REGAN D; SILVER R \& MURRAY TJ. Visual acuity and contrast sensitivity in multiple sclerosis - Hidden visual loss, an auxiliary diagnostic test. Brain 100:563-579, 1977.

11 - MONTEIRO MIR. Sensibilidade ao contraste em portadores de defeitos campimétricos atribuídos a tumores hipofisários. Tese de Doutorado, Faculdade de Medicina da USP, São Paulo. p. 1-106,1992.

12 - SANTOS EF. Avaliação da acuidade visual e sensibilidade ao contraste em trabalhadores expostos a agrotóxicos organofosforados. Tese de Doutorado, Faculdade de Medicina de Ribeirão Preto da USP, Ribeirão Preto. p. 166, 1995.

13 - SAMPLE PA; JUANG PS \& WEINREB RN. Isolating the effects of primary open-angle glaucoma on the contrast sensitivity function. Am J Ophthalmol 112:308-316, 991.

14 - BRETON ME ; WILSONTW; WILSON R; SPAETH GL \& KRUPIN, T. Temporal contrast sensitivity loss in primary open-angle glaucoma and glaucoma suspects. Invest Ophthalmol Vis Sci 32: 2931-2941, 1991.

15 - GREEN DG. Visual acuity, color vision and adaptation. Biol Eye 20:334-336, 1968.

16 - MAINSTER M. Contemporary optics and pathology. Surv Ophthalmol 23:135-142, 1978.

17 - FITZKE AW. Clinical psychophysics. Eye 2: 233-241, 1988. Suppl. 
18 - MILLER D. Glare and Contrast Sensitivity Testing. In: TASMAN W \& JAEGER EA. Duane's: Foundations of clinical Ophthalmology. Revised Edition. Lippincott-Raven, Philadelphia, V. 1, cap. 35, p. 1-19, 1995.

19 - DRAKE MV. Glaucomatous visual loss: field, color and contrast. Int Ophthalmol Clin 30:169-176, 1990.

20 - BRON AJ. Contrast sensitivity changes in ocular hypertension and early glaucoma. Surv Ophthalmol 33: 405-406, 1989. Suppl.

21 - VELTEN IM. Temporal contrast sensitivity with peripheral and central stimulation in glaucoma diagnosis. $\mathrm{Br} \mathbf{J}$ Ophthalmol 83:199-205, 1999.
22 - FRANCE TD \& FRANCE LW. Low-contrast visual acuity cards in pediatric ophthalmology. Graefes Arch Clin Exp Ophthalmol 226:158-160, 1988.

23 - SILVA AC. Avaliação da função da sensibilidade ao contraste em pacientes portadores de glaucoma e hipertensão ocular. Tese de Doutorado. Faculdade de Medicina de Ribeirão Preto da USP, Ribeirão Preto. p. 1-95, 2002.

Recebido para publicação em 22/09/2002

Aprovado para publicação em 30/12/2002 